\title{
Schlafapnoe und COPD - Wenn das Schlechte zum Schlechten kommt
}

\author{
Wenn Schlafapnoe und COPD zusammenkommen, \\ ist die Prognose der Patienten besonders schlecht. \\ Solche sogenannten Overlap-Patienten sollten daher \\ erkannt und entsprechend behandelt werden.
}

Die Kombination der schweren chronisch obstruktiven Lungenerkrankung COPD mit obstruktiver Schlafapnoe (OSA) (Overlap-Syndrom) geht mit einer erhöhten Morbidität und Mortalität einher. Die theoretische Prävalenz des Overlap-Syndroms betrage $1 \%$ in der Allgemeinbevölkerung, berichtete Prof. Winfried Randerath vom Bethanien-Krankenhaus in Solingen auf dem europäischen Lungenkongress in Mailand.

\section{Risiko für Herzrhythmusstörungen und Lungenhochdruck}

Ursache der obstruktiven Schlafapnoe ist eine funktionelle Instabilität der oberen Atemwege. Der pharyngeale Kollaps bei entspannter Muskulatur setzt anatomische und neuromuskuläre Kontrollmechanismen außer Kraft und verschließt die oberen Atemwege teilweise oder ganz (Schwartz AR et al. J Appl Physiol 2006,100:5-6).

Bei COPD-Patienten, meist ältere Menschen mit zahlreichen Komorbiditäten, ist die Prävalenz von OSA deutlich erhöht. Bei gleichzeitigem Vorliegen von COPD und OSA steigt das Risiko für Herzrhythmusstörungen und Lungenhochdruck, die Lebensqualität nimmt ab. Schätzungsweise hat jeder 6. COPD-Patient im fortgeschrittenen Erkrankungsstadium III oder IV nach GOLD gleichzeitig ein OSA. Bei mittelschwerer bis schwerer COPD sollte routinemäßig in einer polysomnografischen Untersuchung nach OSA gefahndet werden, denn eine nächtliche CPAP-Therapie (kontinuierliche Überdruckbeatmung) per Maske verbessert bei den Betroffenen die Schlafqualität, Exazerbationen der COPD und Hospitalisierungen werden vermindert, kardiovaskuläre Todesfälle nehmen unter der Beatmungstherapie ab (Marin JM et al. AJRCCM 2010,182:325-31).

\section{CPAP-Therapie kann versagen}

Bei einem signifikanten Anteil der Overlap-Patienten, vor allem bei jenen mit Übergewicht, schlechter Lungenfunktion, niedrigem $\mathrm{PO}_{2}$, höherem $\mathrm{pCO}_{2}$ im Wachzustand oder längerer Schlafzeit mit $\mathrm{SpO}_{2}$ $<90 \%$, kann die CPAP-Therapie jedoch

Nach einem systematischen Review (Shawon MD et al., Sleep Medicine Reviews 2017,32:58-68) von 21 Beobachtungsstudien ( $>29.000$ Teilnehmer) war die Prävalenz des Overlap-Syndroms bei OSA- und COPD-Patienten stark erhöht (OSA: 7,6-55,7\%) (COPD: 2,9-65,9\%). Overlap-Patienten haben eine ausgeprägte nächtliche Sauerstoff-Unterversorgung $\left(\mathrm{SpO}_{2}<90 \%\right)$ sowie eine schlechtere Schlafqualität im Vergleich zu Patienten mit OSA allein.

Schlafapnoe mit kurzen Atemaussetzern während des Schlafs (bis hin zu einer Minute Dauer), ohne dass der betroffene Patient aufwacht, vermindert die Schlafqualität und die Tagesbefindlichkeit und begünstigt das Auftreten von Sekundenschlaf am Tag. Arousals verhindern zwar das Ersticken. Sie aktivieren aber den Herzschlag, erhöhen den Blutdruck und die Muskelspannung und verflachen das Schlafstadium - alles mit unerwünschten Folgen für die Gesundheit. Schlafapnoe geht darüber hinaus mit unregelmäßigem Schnarchen und oft auch mit Kopfschmerzen beim Erwachen einher. versagen. Hyperkapnie tagsüber und nächtliche Hypoxie sind unabhängige Prädiktoren für ein frühes CPAP-Versagen bei OSA-COPD-Overlap-Patienten (Kuklisova Z et al., Sleep Med 2017,30:139-45).

Eine Langzeit-nicht-invasive-Positiv-Druck-Beatmung (NPPV) kann nach einer Studie bei Patienten mit schwerer stabiler hyperkapnischer COPD das Überleben verbessern (Köhnlein, T et al. Lancet Respir Med 2014,2:698-705). Demnach verllängert eine effektive Senkung des $\mathrm{paCO}_{2}$ durch die NPPV-Therapie das Gesamtüberleben bei Patienten mit schwerer COPD, verbessert die Lungenfunktion und steigert krankheitsspezifische Lebensqualität. Maßgeblich sind dabei die relativ hohen Beatmungsdrücke mit hohen Back-Up-Frequenzen, was einer kontrollierten Beatmung nahe kommt (Magnet F, Atemwegs- und Lungenkrankheiten 2015,4:204-6).

Dagmar Jäger-Becker

Quelle: Best of the ERS/ESRS Sleep and Breathing Conference 2017, European Respiratory Society Congress 2017, Mailand, 9.-13.9.2017 